\title{
Seele auf Kredit
}

Brigitte Boothe

\section{Das Kind als Vorentwurf einer denkenden und fühlenden Person}

Eltern können nicht anders: sie betrachten ihre Kinder als beseelte Wesen. Das ist eine Tatsache zwischen Körper und Geist. Erst im Blick der Eltern auf den Säugling entsteht aus körperlichem Leben geistiges Leben, wird leibliches zur Sprache der Seele. Elterliche Zuschreibung formuliert ein Beziehungsangebot, ein Beziehungsangebot, das Seele im Netz der Beziehungen geltend macht. Seele wird geltend gemacht auf der Basis fundamentaler Kreditierung (Boothe/ Heigl-Evers 1996; Brazelton/Cramer 1990; Grimmer 2000). Ein basaler Zug von Elternschaft ist, den Kindern Kredit zu geben: Das Kind ist für die Eltern der Vorentwurf einer fühlenden und denkenden Person. So sehen sie im Säugling ihresgleichen, interpretieren seine Regungen fortwährend als Äusserungen der Verständigkeit. Sie nehmen das Kind nach seinen späteren Möglichkeiten, sind ihm gewissermassen immer Schritte voraus und bleiben im Verkehr mit dem Kind doch auch in dichtem, direktem Kontakt, bereit, auf $\mathrm{Zu}-$ wendung und Abwendung, Verlangen und Überdruss, Vergnügen und Missbehagen wohlabgestimmt zu antworten (Stern 1985).

\section{Seele hat man nicht - Seele ist das Geschenk des Anderen}

Der Säugling als die lebendige Existenz, mit der Mutter und Vater, Grossmutter und Onkel, Bruder und Neffe intensiv in kommunikativen Kontakt treten, präsentiert sich dem Blick seines Gegenübers in seiner Zukünftigkeit. Man geht mit dem Kind um als mit einem Versprechen, als der Verheissung einer künftig handelnden und denkenden Person. In diesem Sinne gestalten die älteren und erwachsenen Kommunikationspartner die Begegnung mit dem Kind als Vorentwurf späterer dialogischer Partnerschaft. Die spannungsregulierenden, die Orientierung und Kontakt suchenden Regungen und Artikulationen des Kindes erhalten im Dialog mit den erwachsenen Bezugspersonen ihren Platz im kommunikativen Zusammenhang. Der Erwachsene übernimmt im Dialog stellvertretend für das Kind dessen Artikulationen oder führt sie weiter. Mienenspiel und Gestik, Lautlichkeit und Bewegungsrhythmus, Berührung und Handlungsablauf als gemeinsames Tun und Erleben bilden im vielschichtigen Zusammenklang die Wiege, das "Kindbett", der personalen Beziehung in ihrem szenischen Charakter. Hier wurzelt die inszenierende 
Ausdrucks- und Gestaltungsfähigkeit. Sie keimt im kommunikativen Zusammenspiel mit ungleichen Rollenverteilungen. Das Menschenjunge trifft mit Beginn seines Lebens auf Ereignisse, in denen es in vitale Spannung gerät. Das sind körperliche Ereignisse mit vitalem Aufforderungscharakter. Sie vollziehen sich als spannungsvolle Episoden. Blick- und Hautkontakt gehören ebenso dazu wie die Regulierung von Bedürfnisspannungen (Hunger, Durst, Schlafbedürfnis, Ausscheidungs-, Bewegungs-, Zuwendungs- und Artikulationsdrang). In der Begegnung mit derartigen Herausforderungen der kindlichen Vitalität befindet sich das Kind notorisch in der Situation empathisch engagierter Unterstützung. Die Eltern verkehren mit dem Kind nicht als mit einem organischen Funktionsbündel, sondern sie hauchen ihm Seele ein. Damit erweitern und ergänzen sie entlang dem körperlichen Austausch das Bewältigungsund Gestaltungsrepertoire des Kindes. Wenn sie sich dem Kind im Dienst der Regulierung seiner Bedürfnisspannungen nähern, so ereignet sich zugleich zärtliche, spielerische, erregte Kontaktinitiative und die Freude am schmiegsamen Körper und der Anmut des Kindes. So ist der Säugling den Einwirkungen seiner leiblichen und sozialen Umwelt ausgesetzt und zugleich schützenden und liebenden Begleitern anvertraut (Strauss/Buchheim 2002).

\section{Personalisierung}

Der Säugling nimmt Kontakt auf mit der belebten und unbelebten Welt und bedarf zugleich ausgeprägter und dauerhafter VersorgungsSchutz- und Förderungsmassnahmen seitens der primären Bezugspersonen. Allgemein formuliert erfährt das Kind bereits durch seine dargebotene körperliche Präsenz Akzeptanz durch seine primären Bezugspersonen. Sein aktives Lächeln, das es mit etwa zwei Monaten zeigt, wenn es ihrer ansichtig wird, wird gewöhnlich mit Freude begrüsst und beantwortet und festigt Familiarität. Die primären Bezugspersonen stellen sich auf das Neugeborene ein, indem sie es als Person behandeln, als Beziehungspartner mit Rechten. Der kindliche Beziehungspartner geniesst eine Ausnahmestellung. Man billigt ihm erhöhte Aufmerksamkeit und ein Mehr an zärtlicher Betreuung zu, bei gradueller Entlastung von Gegenseitigkeit. Die elterliche Beziehungshaltung dem Kind gegenüber ist auf eingreifende Unterstützung oder unterstützendes Eingreifen sowie begleitende und konturgebende Artikulation des kindlichen Handelns und Erlebens angelegt. Wenn das Kind erschrickt, wenn es staunt, wenn es Anstrengung zeigt, wenn es unter Unlustspannung steht, wenn es jauchzt und gluckst, findet es die bewillkommnende, haltgebende Resonanz des tätigen Gegenübers. 


\section{Brigitte Boothe}

\section{Seele auf Kredit als Teil eines Einbettungsgeschehens}

Gehen wir einen Schritt zurück und betrachten wir die Gabe der Beseelung als Teil einer kulturellen Gepflogenheit, die so selbstverständlich ist, dass sie dem Besprochenwerden entgeht: auf die Bereitschaft und die Leistung der Familienmitglieder, das Kind in eine spezifische familien- und individualgeschichtliche Reihe zu stellen, ihm einen kulturell und individuell vorinterpretierten Ort als national, regional, familiär bestimmtes Geschlechtswesen zuzuweisen, das Kind als familiär zugehörig zu definieren, seine Lebensregungen von Beginn an - ja, bereits im Mutterleib - als sinnvoll, spezifisch und individuell $\mathrm{zu}$ interpretieren sowie eben diesen Lebensregungen zukunftsweisende Bedeutung zu geben. Das Kind erhält eine Geschichte (Historisierung) und wird antizipatorisch als denkende und fühlende Person behandelt (Kreditierung). Man macht es zu einem Wesen an einem bestimmten historischen Ort (Historisierung), verleiht seinen Lebensregungen Sinn und Bedeutung und begreift sie als Anzeichen für künftige Weiterentwicklungen (Kreditierung). Diese ausgeprägte elterliche Zuschreibungstätigkeit hat also das Ziel, das noch unmündige Lebewesen als beseeltes Individuum geltend $\mathrm{zu}$ machen.

\section{Historisierung}

Dass ein menschlicher Neuankömmling einen geschichtlichen Ort erhält, indem man ihn als jüngstes Mitglied einer familiären Gemeinschaft willkommen heisst, ist die positive Normerwartung (Scholz 1994). Die Pointe der Historisierung ist, dass ein Kind heranwächst nicht als Individuum-für-sich-selbst, sondern als Träger von Zuschreibungen, die den Neuankömmling mit einer Vorgeschichte, einer Beziehungsgeschichte und einer Entwicklungsgeschichte einkleiden und den die Eltern so willkommen heissen. Die soziale Aktivität der Zuschreibung von Geschichte erscheint als ein fundamentales Ereignis der Menschwerdung des Einzelnen als Sozialwesen. Die alltäglichen und festlichen Riten der Herstellung von Familiarität modulieren stets das gleiche Thema: Die soziale Produktion von Familiarität entspricht der Herstellung eines Ich-in-Beziehungzum-antwortenden-Gegenüber, eines Ich-als-Teil-eines-Wir, eines Ich-das-zwischen-Du-und-Wir-und-Ihr-unterscheidet:Herstellung spezifischer Abgrenzungen sowie Herstellung spezifischer Verbindung innerhalb einer geschichtlich bestimmten, füreinander engagierten, aufeinander orientierten Gemeinschaft. Historisierung gestaltet sich variantenreich als Fest der Bewillkommnung und Aufnahme des Kindes in den sozialen Weltkreis, etwa durch Geburtsanzeige, Namensgebung, Taufe. Familiäre Akte der Herstellung von Zugehörigkeit sind Legion. 


\section{Aussetzung}

Der Historisierung als komplexer Aktivität sozialisierter Individuen, den menschlichen Neuankömmling per Deklaration und mit Hilfe von Ritualen der Herstellung von Zugehörigkeit familiär einzuwurzeln, steht die Aussetzung gegenüber. Aussetzung kommt der Weigerung oder der Unfähigkeit gleich, dem Kind Zugehörigkeit anzubieten und es in Zugehörigkeit hineinzuziehen. Aussetzung meint ein elterliches Handeln, das darauf zielt, sich des Kindes zu entledigen. Die Extremvariante dieser Aktivität besteht in der Entfernung des Kindes aus elterlicher Obhut mit voller Inkaufnahme des Risikos, dass der Säugling nicht überlebt, das Kind zu schwach ist.

Dennoch ist Aussetzung von direkten oder indirekten Tötungshandlungen zu unterscheiden. Aussetzung des Kindes stellt die Weigerung dar, den Nachwuchs als Teil der eigenen Person, der eigenen Familie, der eigenen Geschichte anzunehmen. Die Inkaufnahme seines Zugrundegehens erfolgt nicht mit dem primären Ziel der kindlichen Vernichtung. Kindstötung liegt somit nicht auf der gleichen Ebene wie Aussetzung. Aussetzung ist vielmehr das Ergebnis ausbleibenden Engagements für das Kindeswohl. Aussetzung ist in dieser Perspektive enger verwandt mit Vernachlässigung als mit Kindesmord. Die Intention der Aussetzung ist nicht, das Kind am Weiterleben zu hindern, sondern dessen Existenz in radikaler Loslösung von der eigenen anzusehen. Während die Tötung des Kindes als Versuch einer Trennung zu werten ist, setzt Aussetzung gerade die Trennung voraus. Oder, um es genauer zu formulieren: Aussetzung ist das Ergebnis niemals hergestellter Knüpfung der symbolischen Nabelschnur. Aussetzung bedeutet radikales Nicht-Annehmen wie im Reich der brutpflegenden Säugetiere vorgebildet - und hat die radikale Verkümmerung des Kindes zur Konsequenz. Elterliches Sich-Kümmern erscheint in dieser Perspektive als aktive und zielgerichtete Orientierung auf das Kind, zielgerichtet im Sinne eines normengeleiteten, hochdifferenzierten Einsatzes, der das Gedeihen des Kindes will, sich am Gedeihen des Kindes bemisst. Wir wollen weniger offensichtliche Fälle betrachten. Wenn "Aussetzung" die Nicht-Beziehung zum Nachwuchs ist, als zu einem geschichtslosen Fremdling ohne Anspruch auf Interesse, so öffnet sich der Blick für fast alltägliche Phänomene, in freilich viel feineren und abgestufteren Varianten. Die bewusste - oft nur flüchtige und vorübergehende - Regung, das Kind nicht als sein eigen Fleisch und Blut anzunehmen, gehört zum Alltag der Elternschaft. Sie artikuliert sich in Wendungen wie: Es ist wie ein Fremdkörper (etwa bei einer Schwangeren); ich schaue es an (das Neugeborene), finde es fremd, bin ganz ratlos; das soll mein Kind sein?; das Kind ist so seltsam, ich verstehe 


\section{Brigitte Boothe}

es nicht, was habe ich mit ihm zu tun? Gewöhnlich sind diese Regungen flüchtig, situationsbedingt, rasch vorübergehend; sie werden meist als sehr unangenehm erlebt, denn woran läge Eltern mehr als am herzlichen Einvernehmen mit und auch an der ungeteilten Hinwendung zum Kind? Weniger flüchtig ist die innere Aussetzungsregung - die sich auch hier nicht als äussere Entledigungshandlung manifestiert - bei depressiven mütterlichen Reaktionen nach der Geburt des Kindes: Die freudige Annäherung an das Kind bleibt aus, das sonst so stark engagierte entzückte allmähliche Kennenlernen findet nicht statt. Zwischen Kind und primärer Bezugsperson bleibt eine Kluft der Kontaktlosigkeit. Aussetzung ist nicht notwendigerweise ein Störungs-, sondern durchaus auch ein reguläres Alltagsphänomen. In diesem Sinn sind alltägliche Aussetzungsreaktionen all jene, bei denen ein Verhalten, eine Reaktion, ein anderer Aspekt des Kindes ausserhalb der Einbettung durch Historisierung bleibt. Es handelt sich hier um Inseln des kindlichen Lebens, die zwischen Kind und primärer Bezugsperson in kein historisierendes Interpretationsmuster fallen. Es sind Bedürfnisregungen, Beziehungsangebote, Verhaltensweisen des Kindes, die von primären Bezugspersonen nicht beachtet, ignoriert, übersehen, nicht gewürdigt oder mit Gleichgültigkeit und Desinteresse gesehen werden, die also keinen Eingang in jenes persönliche Geschichtsbuch finden, das durch historisierende Aktivität der Eltern vom Leben des Kindes entsteht. Freilich hätte ein extremes Mass an elterlicher Bereitschaft, die Lebensregungen des Kindes zu historisieren, den Charakter einer Verfolgung, es bliebe kein freier Spielraum, das Kind wäre in einem Korsett von Zuschreibungen gefangen. Zur Selbstverfügung des Kindes wie später des Jugendlichen gehört der spielerische Umgang mit den erfahrenen Zuschreibungen, deren Anverwandlung wie deren Verfremdung, deren Ersetzung und Verwerfung, die Herstellung neuer Familienromane, sowie die verschiedensten Formen der Selbst-Aussetzung, d.h. spielerischer oder leidenschaftlich engagierter Formen des Sich-Selbst-fremd-Machens, der Verweigerung von Zugehörigkeit zur Familie.

\section{Zurück zur Seele auf Kredit}

Historisierung und Aussetzung sind zu ergänzen durch das antagonistische Paar: Kreditierung und Verweigerung. Unter "Kreditierung" ist die bereits erwähnte elterliche Haltung zu verstehen, die Lebensregungen des Kindes - im Mutterleib, als Neugeborenes, als Kleinkind, sogar auch im späteren Entwicklungsalter - mit einer Entwicklungsaussicht zu verknüpfen, sie als positives Anzeichen für eine weitere Entfaltung in der Zukunft zu sehen. Den Lebensregungen 
des Kindes wird eine Entwicklungsperspektive zugeschrieben. Was das Kind jetzt tut, das gilt den Angehörigen als Versprechen einer Kompetenz, die sich einstweilen nur ahnen lässt oder in ersten, noch einfachen Realisationsformen produziert (Brinich 1982). Die Zuschreibungshandlungen der Kreditierung stellen eine Selbstverständlichkeit des Alltags im Umgang zwischen Erwachsenen in Elternfunktion und Kindern dar und sind bekanntlich nicht allein auf die Eltern-Kind-Beziehung beschränkt (Dennett 1981). Kreditierung ist als Etablierung einer Hoffnungsperspektive in der Kommunikation generell wirksam, Grundlage sowohl ermutigten Lernens, Ausprobierens und Durchhaltens trotz Rückschlags als auch der Illusionsbildung. Die christliche Kultur hat das Bild vom Kind als Verheissung ausgeformt und grossen Einfluss auf die weltliche Kultur ausgeübt. Das Prinzip elterlicher Kreditierung konzipiert das Kind als Werdendes.

Während die soziale Aktivität der Historisierung den Nachwuchs in eine geschichtliche Vergangenheit hinein verankert, verschafft ihm die Kreditierung eine Zukunftsperspektive. Beide stellen Grundformen der Einbettung dar, psychosoziale Grundbedingungen primärer Sozialisation. Beide Grundformen der Einbettung entfalten sich als spezifische Beziehungsstruktur. Der historische "Familienroman« wird also durch einen utopischen »Kindsroman« ergänzt. In beiden Fällen handelt es sich um narrative Strukturen, geflochten aus Dichtung und Wahrheit, kunstreich gefügt aus elterlichen Beziehungsangeboten an das Kind und erwarteten wie interpretierten kindlichen Antworten, später auch aus kindlichen Beziehungsangeboten und erwarteten wie interpretierten elterlichen Antworten.

"Familienroman" und "Kindsroman" besitzen ausserordentliche Suggestionskraft. Sie modellieren Lebensentwürfe des Kindes, Entwürfe kindlichen Lebens, stellen in psychoanalytischer Perspektive, sofern es sich um gefällige Entwürfe handelt, beträchtliche Gratifikationen des Selbstwerts bei allen Beteiligten dar und begründen auf diese Weise ein starkes Motiv, das Erwachsenwerden nicht gerade herbeizusehnen, sondern die Situation des Kindes auszukosten, dem sich der elterliche Blick, die elterliche Bereitschaft, das Kind als Verheissung zu sehen, verschwenderisch zuwendet. Hier bildet sich Ambivalenz aus: Dem von Freud stark beachteten leidenschaftlichen Wunsch, grösser zu werden, um selbst Vater- oder Mutterstelle einzunehmen, steht der Sog des Kind-Bleibens gegenüber - ewige Verheissung, ewige Hoffnung, ewiger Neuanfang, ewige Künftigkeit. Familienroman und Kindsroman sind prägende Modelle eines Lebens, das auf diese Weise Dimensionen des Vergangenen und des $\mathrm{Zu}-$ künftigen erhält. Kreditierung basiert auf der Möglichkeit eines we- 


\section{Brigitte Boothe}

nigstens abschnittsweise gemeinsamen Lebenswegs und auf der Sicherheit des Erwachsenen, über Ressourcen zur Förderung des kindlichen Potentials zu verfügen. Ist beides nicht gegeben, so stellt sich die um Kreditierung zentrierte Form der asymmetrischen Kommunikation gar nicht erst ein oder versandet. Das Kind lallt, das Gesicht mit freudigem Ausdruck der Mutter zugewendet, die mütterliche Zuhörerin ruft entzückt: $O$, du sagst ja schon Mama! Dieser prototypische Akt der Kreditierung bindet sich an die mütterliche Bereitschaft, die noch wenig artikulierten Äusserungen des Kindes in bezug auf ein Künftiges zu hören, ein Künftiges, das auch für sie selbst von emotionalem Interesse ist. Sie bettet die Lebensäusserung also ein in ein von ihr vorentworfenes emotionales Beziehungsnetz, in der selbstverständlichen Bereitschaft, die Äusserungen des Kindes weiterhin in der entsprechenden Art aufzunehmen (Dennett 1981) und ihm den Charakter von Kompetenz und Vermögen zuzusprechen und aktiv zu fördern. Dies ist im Alltag leicht zu beobachten. Die entzückte Mutter, die sich vom Kind aktiv mit dem auratischen Namen Mama angesprochen weiss, beginnt sogleich ein Spiel der Amplifikation: das Wort selbst in artikulierter Betonung wiederholend, in melodischem Singsang, etc. Die Mutter greift die vielleicht zufälligen kindlichen Lautäusserungen auf, führt sie weiter, baut sie aus, ist dabei mit dem Kind in engem körperlichem Kontakt.

\section{Verweigerung}

Diese Spiele bleiben aus, wenn das Kind als Fremdes erlebt wird, müssen zwangsläufig ausbleiben, weil sich unter den Bedingungen des Fremdseins die Selbstverständlichkeit und Fraglosigkeit mentaler Zuschreibungsaktivität normalerweise minimiert. Es geht um eine Form des Kontakts mit dem Kind, der die Zukunftsperspektive ausspart. Die versorgenden oder beaufsichtigenden Personen ignorieren die Möglichkeiten des Kindes; sie tun das, wenn sie keine engagierte Verbindung zum Kind unterhalten. Die engagierte Verbindung zum Kind bindet sich zwar nicht an reale Elternschaft, aber es bedarf einer individuellen und um die Zukunft des Kindes besorgten Beziehung. Unter institutionellen Bedingungen ist eine derartige Beziehung kaum wahrscheinlich; und dies ist durchaus keine Frage schuldhaften Vergehens oder persönlichen Desinteresses der Betreuungsperson am Kind. Ausbleibende oder verminderte Historisierung sowie ausbleibende oder verminderte Kreditierung bedeuten eine primäre Ausgrenzung des Kindes aus der Kommunikationsgemeinschaft. Das Kind ist dann nicht in eine familiäre Gruppierung eingebettet. Seine Existenz ist prekär, randständig, unintegriert. Die 
Begegnung mit dem Kind hat ausserhalb der einbettenden Kommunikation keine Sprache.

Das Kind in der geschichtlichen Reihe auf der einen Seite, das namenlose Ausgesetzte auf der anderen; das Kind, dessen Lebensregungen als Verheissungen begrüsst werden einerseits, dasjenige, dem kein liebevoll zukunfts- und hoffnungsbeflügelter Blick gilt andererseits: Hier werden auf einer fundamentalen Ebene der Herstellung von Beziehungen zwischen Kind und Betreuungspersonen Entwicklungslinien vorgezeichnet, wird die Grundlage dafür geschaffen, ob ein Menschenjunges im Prozess der Reifung von einer Basis der Zugehörigkeit ausgehen kann, ob es sich als Individuum an einem geschichtlichen Ort versteht, als Person, das Resonanz und Antwort beim Gegenüber hervorruft, oder als marginale Existenz in einer unwirtlichen Welt, die undurchdringlich bleibt und sich verschliesst.

\section{Blick zurück}

Das Kind ist existentiell angewiesen auf einfühlende, kommentierende, Bedeutung gebende Begleitung durch sprachmächtige, sozialisierte Bezugspersonen. Aktives Bezogensein und einflussträchtige Regsamkeit des Kindes bilden eine Arena der Expressivität, in der die sprachmächtigen, sozialisierten Individuen versorgend, sichernd, steuernd, kontrollierend und zärtlich handeln (Fogel 1993). Der Säugling legt in die Welt hinein eine vitale Spur, eine deutungsbedürftige Spur. Die Erwachsenen geben in fortwährender spontaner Deutung den Lebensregungen des Kindes Sinn.

Im Rahmen der dichten dialogischen Bezogenheit von Kind und primären Beziehungspartnern findet die Platzzuweisung des Kindes oder seine Familiarisierung im sozialen Raum statt. Seele entsteht als humane Tatsache, wird im kommunikativen Zusammenspiel etabliert. Die Lebensregungen des Kindes werden im Dialog so aufgenommen, dass der Säugling als Familienmitglied eingebettet wird. Seine Lebensregungen werden bereits im Mutterleib als sinnvoll, spezifisch und individuell interpretiert. In der Perspektive der Einbettung ist das Kind Gegenstand der Historisierung und Kreditierung: Man macht es zu einer Person an einem bestimmten historischen Ort, verleiht seinen Lebensregungen Sinn und Bedeutung und begreift sie als Anzeichen für künftige Weiterentwicklung. Diese ausgeprägte elterliche Zuschreibungstätigkeit mit dem Ziel der Einbettung des Kindes geschieht im Gespräch über das Kind und im Gespräch mit dem Kind (Boothe \& Heigl-Evers 1996: 124 ff). Historisierung stellt Zugehörigkeit her. Aufgrund von Historisierung wird der Neuling im Leben mit einer Vorgeschichte, einer Bezie- 


\section{Brigitte Boothe}

hungsgeschichte und einer Entwicklungsgeschichte eingekleidet. Die Eltern finden ihn in dieser Ausstattung kenntlich und heissen ihn willkommen. Die soziale Aktivität der Zuschreibung von Geschichte erscheint als ein fundamentales Ereignis der Menschwerdung des einzelnen. Seine Inszenierung gestaltet sich variantenreich als Fest der Bewillkommnung und Aufnahme des Kindes. Es vollzieht sich das Wunder der Transzendenz von der Biologie zur Seele.

\section{Literatur}

Boothe, B./Heigl-Evers, A. (1996), Psychoanalyse der frühen weiblichen Entwicklung, München.

Brazelton, T.B./Cramer, B.G. (1990), Die frühe Bindung, Stuttgart.

Brinich, P.M. (1982), »Rituals and meanings: The emergence of motherchild communication", Psychoanalytic Study of the Child 37, 3-15

Cramer, B. (1991), Frühe Erwartungen. Unsichtbare Bindungen zwischen Mutter und Kind, München.

Dennett, D. (1981), »Intentionale Systeme«, in: Bieri, P. (Hg.), Analytische Philosophie des Geistes, Meisenheim, 162-183.

Fogel, A. (1993), Developing through relationships, Hertfordshire.

Grimmer, B. (2000), "Kreditierung in einer psychoanalytisch orientierten Psychotherapie«, Psychotherapie und Sozialwissenschaft 4, $256-277$.

Scholz, G. (1994), Die Konstruktion des Kindes, Opladen.

Stern, D. (1985), The interpersonal world of the infant. A view from psychoanalysis and developmetnal psychology, New York.

Strauss, B./Buchheim, A. (2002), »Bindung und Trennung: Zum Verhältnis von Bindungstheorie und Psychoanalyse«, Psychotherapie und Sozialwissenschaft 2, $138-152$.

- Dr. Brigitte Boothe ist Professorin für Klinische Psychologie, Psychotherapie und Psychoanalyse an der Universität Zürich. 